\title{
Finite element based design optimization of WENDELSTEIN 7-X divertor components under high heat flux loading
}

\author{
A. Plankensteiner ${ }^{\mathrm{a}, *}$, A. Leuprecht ${ }^{\mathrm{a}}, \mathrm{B}$. Schedler ${ }^{\mathrm{a}}$, \\ K.-H. Scheiber ${ }^{a}$, H. Greuner ${ }^{b}$ \\ ${ }^{a}$ PLANSEE SE, A-6600 Reutte, Austria \\ ${ }^{\mathrm{b}}$ Max Planck Institute for Plasma Physics, Garching, Germany
}

\begin{abstract}
In the divertor of the nuclear fusion experiment WENDELSTEIN 7-X (W7-X) plasma facing high heat flux target elements have to withstand severe loading conditions. The thermally induced mechanical stressing turns out to be most critical with respect to lifetime predictions of the target elements. Therefore, different design variants of those CFC flat tile armoured high heat flux components have been analysed via the finite element package ABAQUS aiming at derivation of an optimized component design under high heat flux conditions. The investigated design variants comprise also promising alterations in the cooling channel design and castellation of the CFC flat tiles which, however, from a system integration and manufacturing standpoint of view, respectively, are evaluated to be critical. Therefore, the numerical study as presented here mainly comprises a reference variant that is comparatively studied with a variant incorporating a bi-layer-type AMC-Cu/OF-Cu interlayer at the CFC/Cuinterface. The thermo-mechanical material characteristics are accounted for in the finite element models with elastic-plastic properties being assigned to the metallic sections $\mathrm{CuCrZr}$, AMC-Cu and OF-Cu, respectively, and orthotropic nonlinear-elastic properties being used for the CFC sections. The calculated temporal and spatial evolution of temperatures, stresses, and strains for the individual design variants are evaluated with special attention being paid to stress measures, plastic strains, and damage parameters indicating the risk of failure of CFC and the CFC/Cu-interface, respectively. This way the finite element analysis allows to numerically derive an optimized design variant within the framework of expected operating conditions in W7-X.

Keywords: WENDELSTEIN 7-X; Divertor; Plasma facing components; Finite element analysis
\end{abstract}

* Corresponding author. Tel.: +43 5672600 2974;

fax: +435672600 501 .

E-mail address: ap@plansee.com (A. Plankensteiner).

\section{Introduction}

The aim of the WENDELSTEIN 7-X divertor target elements is the heat transfer from the plasma facing 


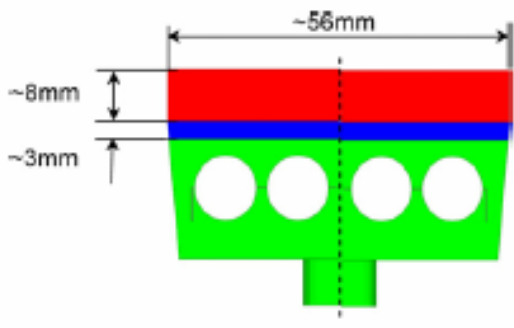

Fig. 1. Sketch of a cross section of a W7-X divertor target element including typical principal dimensions of the cross section and colour coding for the CFC section (red), the AMC-Cu-section (blue), and the CuCrZr-section (green), respectively.

surface approaching $1000^{\circ} \mathrm{C}$ and above to the cooling channel with coolant temperatures between 20 and $80{ }^{\circ} \mathrm{C}$. The target element must allow the handling of applied heat loads of $10 \mathrm{MW} / \mathrm{m}^{2}$ and above. All other aspects such as the thermally induced mechanical stressing of the target element are of artificial nature and, therefore, may be touched only with the heat transfer performance influencing design parameters being unchanged. To meet such extreme thermal boundary conditions, a compound design is employed introducing carbon- and copper-based materials. The plasma facing sections incorporate CFC (carbon fibre reinforced carbon composite) and the heat sink itself incorporates CuCrZr (a precipitation hardened Cualloy), see Fig. 1. This, however, introduces a rather extreme combination of high contrasts in thermal and mechanical material properties of CFC and CuCrZr, high overall height to width ratios, and large absolute temperature differences and steep temperature gradients, respectively. Therefore, an interlayer realized via active metal casting of copper (AMC-Cu) [1] is introduced.
Generally speaking this AMC-Cu-interlayer acts as a transition zone for normal and shear stresses and strains, respectively, this way behaving like an interphase rather than a sharply bounded interface, see Fig. 2. An optimization of the reference design is required due to the relatively high failure rate during high heat flux testing [3] for 20 pre-series target elements that make use of the reference design. Beside the other design parameters (compare Section 2.1) the interlayer design and material characteristics introduce additional design variables that can be adjusted correspondingly. This is done by a finite element based modelling and analysis approach as described below.

\section{Finite element based modeling and simulation approach}

The presented finite element based approach aims at calculating, i.e. predicting, temperatures as well as stresses and strains and corresponding measures, respectively, at a heat flux loading of $10.5 \mathrm{MW} / \mathrm{m}^{2}$. Detailed inspection of the stressing is done for the target element with special attention being paid to the $\mathrm{CFC} / \mathrm{Cu}$-interface. The reference design variant and the design proposal are evaluated and dependencies between design parameters, material properties, and solution parameters (mainly the stressing to the interface) are extracted, thus allowing to evaluate the design proposal. The commercial finite element suite ABAQUS is used for modelling and analysis [2].

\subsection{Investigated design variants, geometry, symmetry conditions}

The divertor target element under consideration is a full-scale target element with $250 \mathrm{~mm}$ in length and covers 10 CFC flat tiles. Symmetry conditions are adopted in order to reduce modelling efforts and CPU
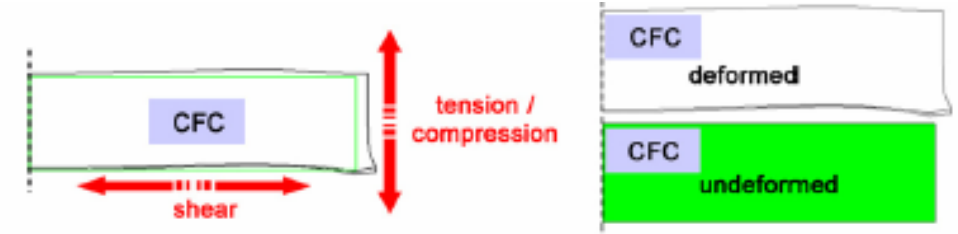

Fig. 2. Sketch of an undeformed (white) and deformed (green) half cross section of the CFC section (other material sections are dismissed) due to heat flux loading indicating the simultaneous appearance of shear stresses and normal stresses at the $\mathrm{CFC} / \mathrm{Cu}$-interface. 

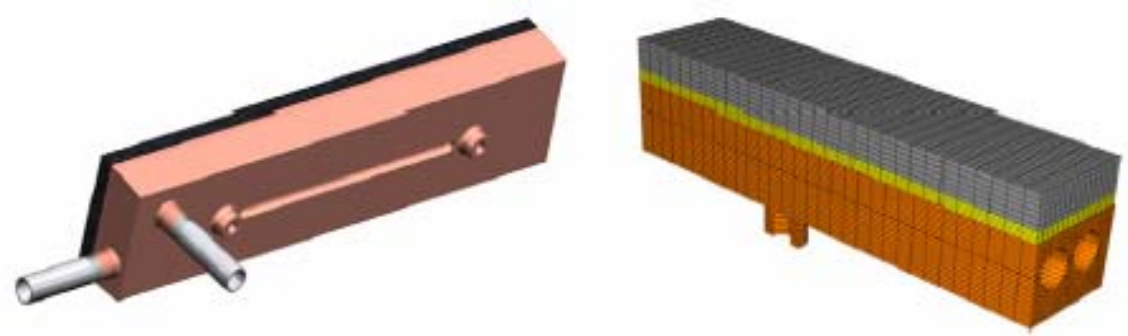

Fig. 3. Sketch of the investigated W7-X full scale target element with $250 \mathrm{~mm}$ in length comprising 10 CFC flat tiles (left) and view of the corresponding 3D FEM model of one quarter of the full target element by making use of symmetry conditions for geometry, loading, and material definitions, respectively (right).

time, see Fig. 3. Different design variants (compare Fig. 4) have been evaluated incorporating:

- Castellation of CFC sections (up to 19 transverse and 8 lengthwise slots for the overall target element), - half width of the component, - $90^{\circ}$-shift of principal material axes of CFC (needling versus Ex-PAN),

- slot at free surface of AMC-Cu-interlayer,

- modified cooling channel geometry (quadratic versus circular),

- ligament thickness between cooling channel and CFC section,

- bi-layer of $\mathrm{AMC}-\mathrm{Cu}$ and $\mathrm{OF}-\mathrm{Cu}$ at the $\mathrm{CFC} / \mathrm{Cu}$ interface,

- and combinations of above.

However, manufacturing aspects, demanding cost aspects as well as the fixed cooling parameters of the W7-X divertor govern the definition of design variables for the target element itself. The proposed bi-layer type AMC-Cu/OF-Cu interlayer at the CFC/Cu-interface together with a $90^{\circ}$-shift of needling versus Ex-PAN material axes represents a design proposal that is in accordance with the above requirements and, therefore, is investigated in the present work comparatively with the reference design the latter representing the original design of the pre-series testing elements.

\subsection{Loading conditions}

The thermal loading is due to a surface heat flux acting continuously for $15 \mathrm{~s}$ on the CFC plasma facing surface ranging from a power density of $10.5 \mathrm{MW} / \mathrm{m}^{2}$ at the half length of the component down to $1 \mathrm{MW} / \mathrm{m}^{2}$ at the free ends, compare [3], and is subsequently followed by a cooling downphase. Surface film conditions are applied at the surface of the cooling channel with a film coefficient ranging from 75,000 $\mathrm{W} /\left(\mathrm{m}^{2} \mathrm{~K}\right)$ at a surface temperature of $60{ }^{\circ} \mathrm{C}$ to 110,000 $\mathrm{W} /\left(\mathrm{m}^{2} \mathrm{~K}\right)$ at $260{ }^{\circ} \mathrm{C}$ [4]. The coolant temperature is assumed to beconstant at $20{ }^{\circ} \mathrm{C}$ and a constant coolant pressure of 10 bar is accounted for [4].

\subsection{Initial and boundary conditions, contact definitions}

The initial temperature of the model is defined to be $20{ }^{\circ} \mathrm{C}$ [4] and the heat flux is applied instantaneously. The displacements at the support of the component are fixed in order to avoid rigid body motion. At
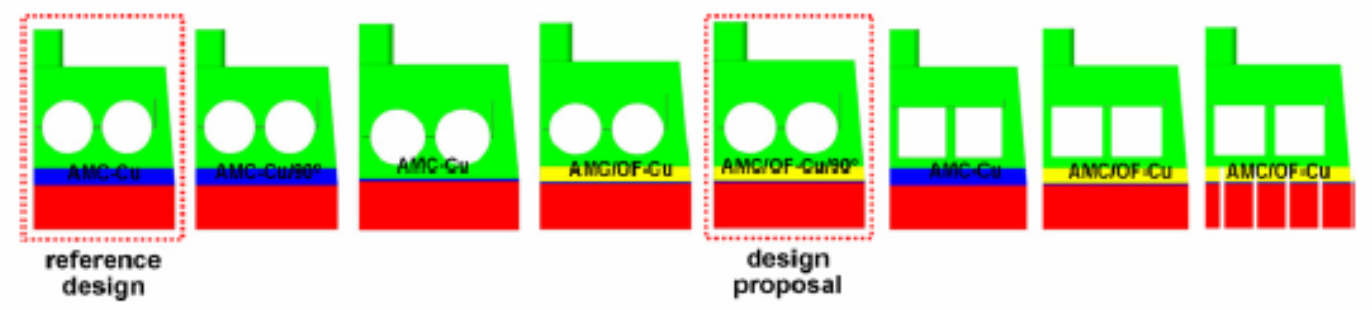

Fig. 4. Excerpt of sketches of investigated design variants with the marked left representing the reference design and the marked right representing the proposed design, respectively. Variants from left to right are listed in ascending order with respect to their potential in stress reduction at the $\mathrm{CFC} / \mathrm{Cu}$-interface region. 


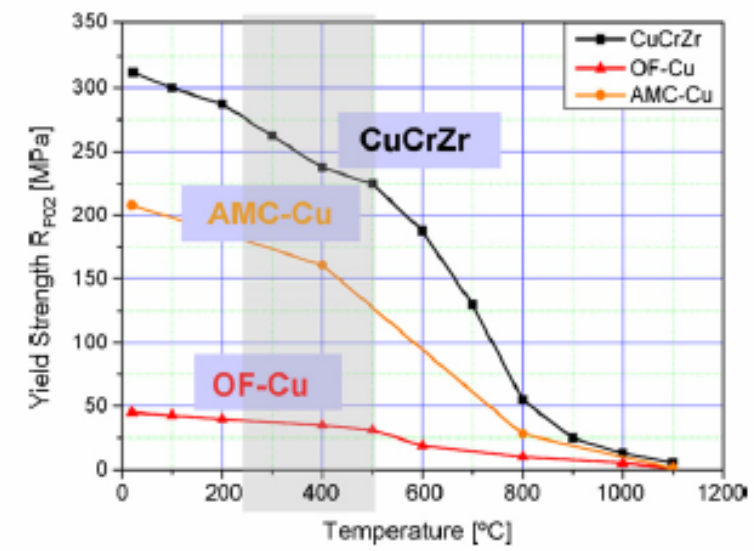

Fig. 5. Measured temperature dependent yield strength behaviour of the adopted metallic materials as used in the finite element analyses of the target element. The grey scale background approximately indicates the acting temperature regime in the interlayer section of the target element under high heat flux loading.

the $\mathrm{CFC} / \mathrm{Cu}$-interface perfect contact conditions are assumed, thus allowing to transfer shear and normal stresses without potential appearance of sliding or normal decohesion. Initial stresses resulting from the last heat treatment cycle of the manufacturing process are accounted for [4].

\subsection{Material definitions}

Individual material definitions are adopted for the metallic and the CFC sections, respectively.

\subsubsection{AMC-Cu, OF-Cu, $\mathrm{CuCrZr}$}

Temperature dependent thermo-elasto-plastic isotropic material descriptions are used for all metallic

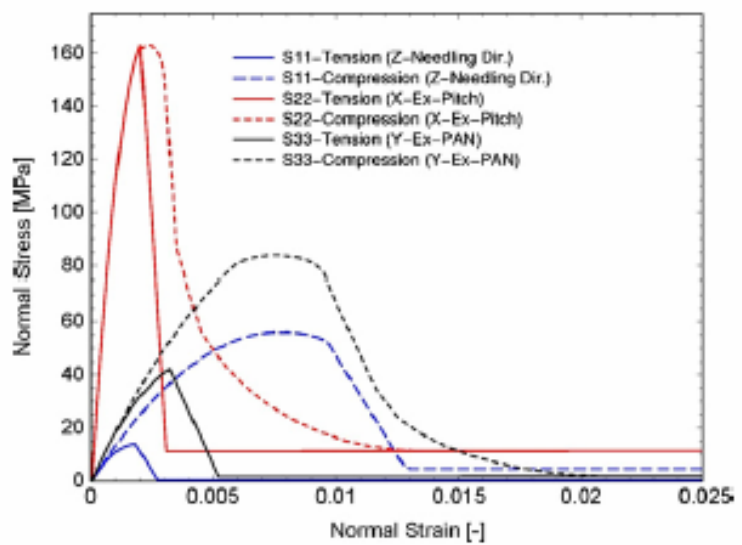

Fig. 6. Uniaxial stress-strain characteristics of CFC grade NB31 according to pilot production as captured by the user defined CFC material model (ABAQUS UMAT) and accounting for the individual behaviour in the individual principal material directions $\mathrm{X}$ (Ex-Pitch, red lines), Y (Ex-PAN, black lines), Z (needling, blue lines) in the tensile (solid lines) and compressive regime (dashed lines), respectively.

sections. For the interlayer as well as for the heat sink, the plastic properties are accounting for the material configurations as they are appearing in the target element in the operational state, compare Fig. 5, and are modelled by multi-linear stress-strain characteristics.

\subsection{2. $C F C-N B 31$}

For the CFC grade NB31 [5] thermo-elastic orthotropic properties are adopted including nonlinear elasticity and progressive damage. A phenomenological constitutive material law is, therefore, developed including evolution equations for internal variables in
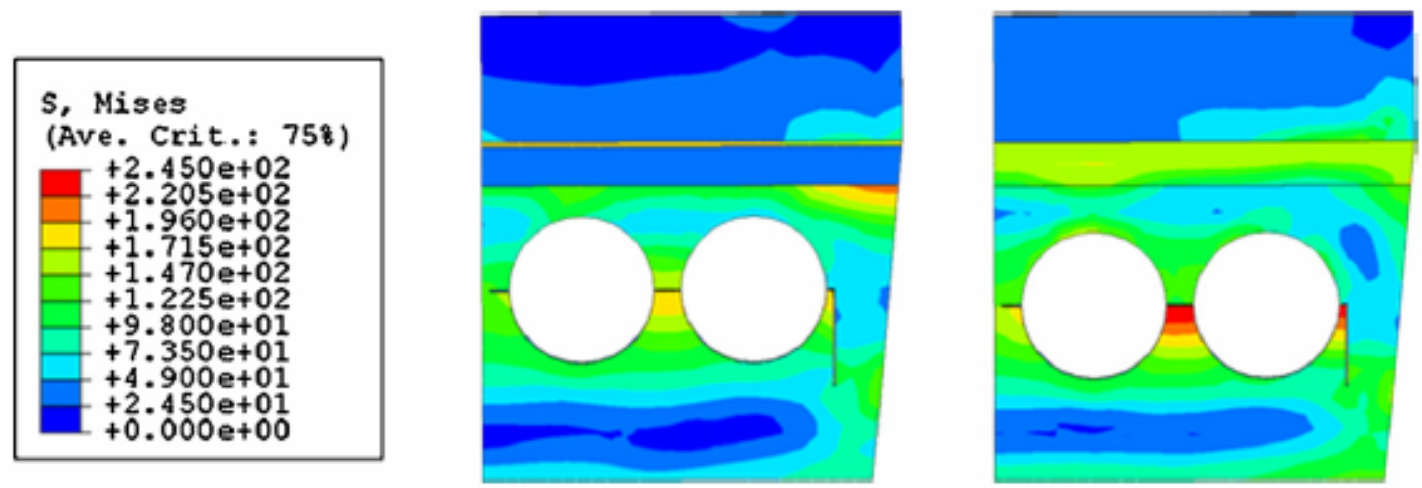

Fig. 7. Calculated distributions of the von Mises stresses [MPa] (MISES) in the cross section at half length of the component at the end of heat flux loading for the design proposal (left) and the reference design (right). 

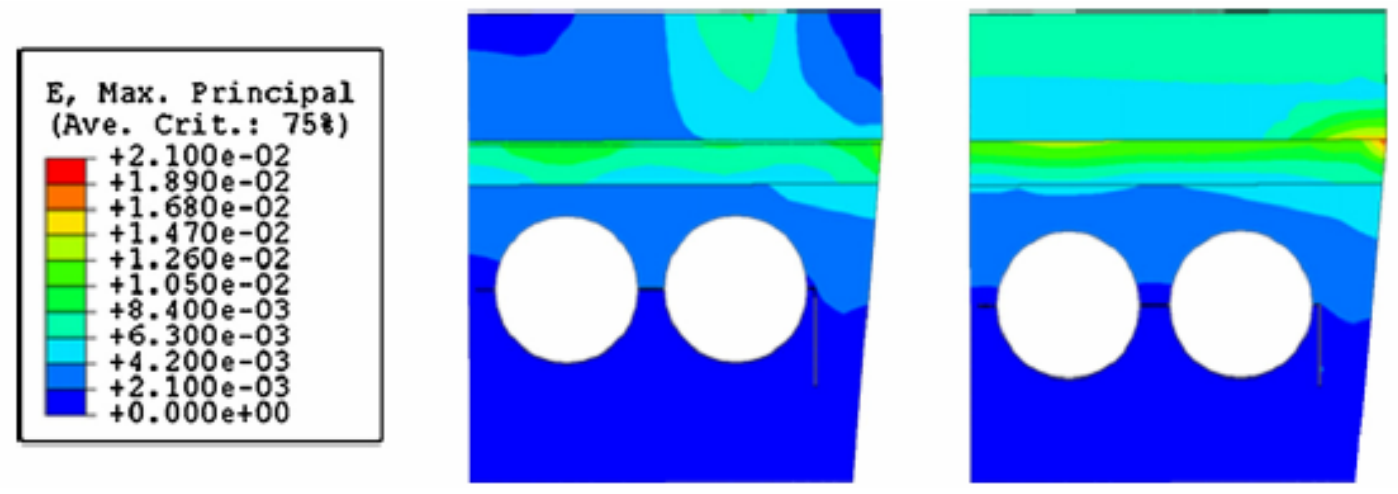

Fig. 8. Calculated distributions of the maximum principal total strains [1] (E, Max. Principal) in the cross section at half length of the component at the end of heat flux loading for the design proposal (left) and the reference design (right). Note that the directions of the maximum principal strains not necessarily correspond to the in-plane directions of the cross-section of the component.

terms of damage relevant parameters describing the CFC behaviour in the total normal stress-strain range, see Fig. 6. Similar descriptions exist for the shear stress versus shear strain behaviour in the individual principal material planes. Unless no localization effects are to be considered and no macroscopic cracks are propagating through the material, the homogeneous degradation of the material may be described within the framework of continuum damage mechanics. In particular, linear relations between individual normal strain components and scalar-type normal damage parameters for tensile and compressive loading, respectively, are introduced in order to account for loss of initial (i.e. virgin) normal stiffness upon loading and subsequent unloading. The same applies to shear loading where individual shear strain components are related linearly to the corresponding scalar-type shear damage parameters, thus accounting for the loss of shear stiffness. A principal hypothesis relies in the fact that the damage behaviour has to be assumed to be uncoupled for the principal material axes and principal material planes, respectively. Coupled damage descriptions, i.e. the introduction of damage parameters of tensorial type, need more sophisticated test setups than those available today which exceed the commonly adopted uniaxial tensile and compression tests, respectively, as well as simple shear tests by far. Finally, it has to be noted that the individual damage parameters progress towards the value of one the latter corresponding to the fully damaged material state, i.e. the fully disrupted material configuration.

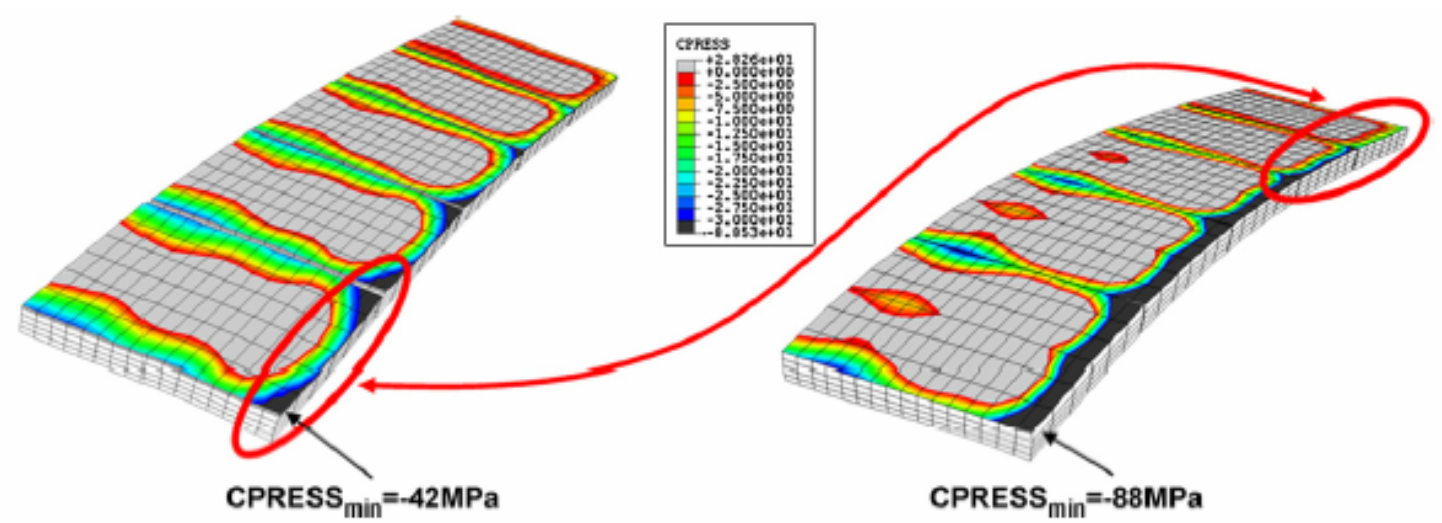

Fig. 9. View on the interlayer at the CFC/Cu-interface (perspectively closer means centre CFC tiles facing maximum heat flux; other material sections are dismissed; deformations scaled by a factor of 25) for the design proposal (AMC-Cu/OF-Cu, left) and the reference design (AMC-Cu, right): Calculated distributions of the normal component of the interface traction vector [MPa] (CPRESS) at the end of heat flux loading (note that negative values for CPRESS mean tensile loading). 

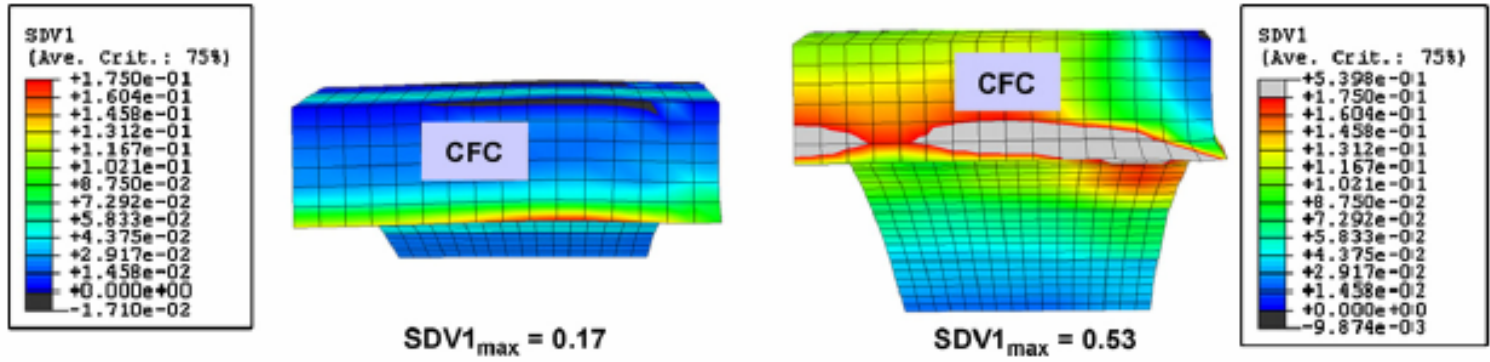

Fig. 10. Front view on the CFC sections (perspectively closer means centre CFC tile facing maximum heat flux; other material sections are dismissed; deformations scaled by a factor of 25) for the design proposal (left) and the reference design (right): Calculated distributions of the solution dependent variable [1] (SDV1) relevant for damage in normal, i.e. high heat flux, direction at the end of heat flux loading.

\section{Discussion of simulation results}

The temporal and spatial evolution of temperatures, stresses and strains are calculated for the reference design (incorporating an AMC-Cu-interlayer) as well as the design proposal (incorporating a bi-layer-type AMC-Cu/OF-Cu-interlayer at the $\mathrm{CFC} / \mathrm{Cu}$-interface) and are evaluated for the CFC sections in terms of maximum principal stresses, damage variables as well as total, elastic and thermal principal strains, and for the metallic sections in terms of von Mises equivalent stresses, equivalent plastic strains as well as total, elastic and thermal principal strains1. In case of the reference design maximum temperatures of $989^{\circ} \mathrm{C}$ are to be expected at the CFC top surface, whereas the proposed design is heated up to $849{ }^{\circ} \mathrm{C}^{2}$. This difference of $140{ }^{\circ} \mathrm{C}$ is decreasing to $110{ }^{\circ} \mathrm{C}$ at the free edge of the $\mathrm{CFC} / \mathrm{Cu}$-interface and shows a significant influence on the stressing and straining of the materials. For instance, the von Mises stress distribution at the end of the heat flux loading is shown in Fig. 7 for both of the design variants with considerably lower stresses to be found in the design proposal. As easily can be seen from Fig. 8 this has also a pronounced influence on the strain- ing, e.g. at the end of the heat flux

\footnotetext{
1 A first step towards verification of the predicted thermal and mechanical results with respect to experimental findings at the GLADIS high heat flux testing facility can be found in Ref. [2].

${ }^{2}$ Note that these temperature results are based on the modelling assumptions which are focusing on the nominal situation with respect to material properties, loading conditions as well as initial and boundary conditions, respectively (compare Sections 2.2-2.4). In particular, a temperature increase may be possible due to a potential shift in material parameters during the manufacturing and application.
}

Loading: whereas, the reference design shows maximum total strains of $2.1 \%$ in the interlayer and $1.6 \%$ in the CFC section, the design proposal shows maximum total strains of $1.1 \%$ in the interlayer and $0.5 \%$ in the CFC section, compare Fig. 8. It is worth noting that these maximum levels of total strain are detected at the free edge of the CFC/Cu-interface which is the most important region of interest with respect to potential interface failure. Note also that for the reference design the thermal contribution on the total strain in the CFC sections is much less than the mechanical contribution. Additionally, for the reference design much higher mechanical straining is predicted compared to the design proposal. The predicted thermal strains for the design proposal are slightly lower than those for the reference design. The plastic straining of the bi-layer-type interface is more pronounced than for the monolayer-type AMCCu interlayer, thisway introducing more compliance in the interaction between the CFC tiles and the heat sink. Therefore, the maximum tensile interface stresses at the free edge of the $\mathrm{CFC} / \mathrm{Cu}$-interface are decreased by a factor of two which may be evaluated as a safe operational condition from an empirical standpoint of view, compare Fig. 9. In particular, for the design proposal, the maximum tensile stresses at the critical free edge of the $\mathrm{CFC} / \mathrm{Cu}$ interface at the $10.5 \mathrm{MW} / \mathrm{m}^{2} \mathrm{HHF}$ loaded CFC tiles are similar to those of the much less loaded CFC tiles in the reference design located at or close to the component's free ends. For both design variants the maximum tensile interfaces stresses appear at half length of the component with values of 42MPa for the design proposal that are less than half of those of the reference design (88MPa). The optimized stress situation 
also results into much less pronounced accumulated damage in the CFC sections. For instance, the accumulated damage due to normal stresses in high heat flux direction can be reduced significantly and, even more important, the maximum values are no longer appearing at the free edge of the $\mathrm{CFC} / \mathrm{Cu}$ interface, compare Fig. 10. In particular, a loss of about $17 \%$ of the stiffness in high heat flux direction is predicted for the CFC material in the design proposal, whereas for the reference design the CFC material looses about $53 \%$ of the initial stiffness.

\section{Conclusions}

The choice of an optimum design variant depends on the potential for decreasing operational temperatures and the minimization of stressing and straining of the component during heat loading and subsequent cooling down, respectively, and the applicability from a manufacturing as well as application standpoint of view. Although, promising design variants including castellation of the CFC flat tiles into subsections, target elements of half of total width, and rectangular cooling channels that are positioned closer to the CFC/Cuinterface are out of focus here, the choice of a bi-layer AMC-Cu/OF-Cu-interlayer at the $\mathrm{CFC} / \mathrm{Cu}$ interface together with material axes being switched (needling versus Ex-PAN) reduces considerably the stressing and straining during the application. The benefit in stress household origins form lowered temperatures together with increased plastic straining compliance in the interlayer. This benefit is predicted at about $50 \%$ reduction in stress levels at the $\mathrm{CFC} / \mathrm{Cu}-$ interface. The manufacturing is set up for upcoming target elements making use of the above design proposal and will be high heat flux tested subsequently.

\section{References}

[1] F. Rainer, N. Reheis, Method for setting-up a cooling system, EP 0663 670, 1994.

[2] ABAQUS/Standard, V6.5, Abaqus Inc., Providence, RI, 2005.

[3] H. Greuner, B. Boeswirth, J. Boscary, A. Plankensteiner, B. Schedler, High heat flux tests of the WENDELSTEIN 7-X preseries target elements, Fusion Eng. Design 82 (2007) 1713- 1719.

[4] H. Greuner, A. Herrmann, H. Renner, P. Chappuis, R. Mitteau, Development of divertor targets for WENDELSTEIN 7-X, in: Proceedings of 20th SOFT, vol. 1, Marseille, France, 1998, pp. 249-252.

[5] P. Majerus, J. Compan, D. Pitzer, M. Roedig, Measurement of physical and mechanical properties of CFC delivered by SNECMA: Final Report, EFDA Task TW2-TVM-CFCQ2, 2004. 